\title{
Advancing teaching opportunities through pre-commercial photonic devices
}

\section{Marko Slusarczuk}

Marko M. G. Slusarczuk, "Advancing teaching opportunities through precommercial photonic devices," Proc. SPIE 9665, Tenth International Topical Meeting on Education and Training in Optics and Photonics, 96650E (3 June 2007); doi: 10.1117/12.2207513

SDIE Event: Tenth International Topical Meeting on Education and Training in Optics and Photonics, 2007, Ottawa, Ontario, Canada 


\title{
Advancing teaching opportunities through pre-commercial photonic devices
}

\author{
Marko M. G. Slusarczuk \\ Optoelectronics Industry Development Association, 1133 Connecticut Ave. N. W., Suite 600, Washington DC 20036 \\ 202-785-4425marko@oida.org
}

\begin{abstract}
The Photonics Technology Access Program [PTAP] provides academic researchers with pre-commercial photonic devices. Since one of the goals of PTAP is to promote teaching, the program has developed several approaches to expand teaching opportunities with the processes used to provide the devices.
\end{abstract}

\section{Introduction}

In July 2002, the United States Government initiated a program whose mission is to provide pre-commercial, stateof-the-art photonic devices to universities for teaching and research. The National Science Foundation [NSF] and the Defense Advanced Research Projects Agency [DARPA] sponsor the Photonics Technology Access Program [PTAP]. The Optoelectronics Industry Development Association [OIDA] administers PTAP and acts as the broker between industry and the universities.

The rationale behind PTAP is that in a rapidly changing technical field, if researchers have to acquire devices on the commercial market, by the time their research is completed and published, the next generation of devices is already on the market. Providing researchers access to devices before they are available on the market, improves the quality and timeliness of their research product. Furthermore, students gain experience with leading-edge technology, enhancing the quality of teaching. Lastly, the program creates and strengthens relationships between faculty and industry. This is particularly important for young faculty who may not have yet developed strong industry connections, and faculty at schools with lesser-known photonic programs.

A second element of PTAP is to act as an "angel investor" and provide seed funding for new ideas. Often a professor or student has an innovative idea but no funds to acquire the devices to test it. Clearly, they can submit proposals to one of the funding agencies, but without some hard data, the proposal may not receive funding. PTAP can supply a device that will enable the researcher to perform some preliminary experiments and obtain data that improve the quality of proposals. This provides a benefit to both the funding agencies, which get higher quality proposals, and researchers, whose odds of success improve. Since PTAP specifically does not provide funding for the research itself, professors must pursue some other source of funding for the actual work.

\section{Evolution of teaching opportunities}

Initially PTAP accomplished its teaching objectives simply by providing novel devices. The process as originally envisaged is relatively straightforward. PTAP would solicit and competitively evaluate brief proposals that request devices. Once the reviewers identify the proposals selected for funding, PTAP notified the researcher and then worked with him or her to identify an appropriate source of device. PTAP would order the device and the vendor would deliver it directly to the researcher and bill PTAP.

\subsection{The first innovation - graduate students as proposal authors}

After the first few competitions, we realized that the true stakeholders in the outcome of the solicitation were the graduate students. They are the ones whose lab work and theses depend on their ability to obtain the necessary materials. Furthermore, given the funding situation today, faculty has to focus on obtaining grants that provide more resources than just an interesting device. To deal with these realities PTAP changed its guidelines to encourage proposals written by students, under faculty supervision.

The attractiveness of having students write the proposals was two-fold. First, for many students this would be the first opportunity to write a proposal that could affect the outcome of their research. We viewed the exercise a

Tenth International Topical Meeting on Education and Training in Optics and Photonics, edited by Marc Nantel, Proc. of SPIE Vol. 9665, 96650E · (c) 2007 SPIE, OSA, IEEE, ICO doi: $10.1117 / 12.2207513$ 
teaching experience, and by requiring the faculty member to sign off on the proposal, the students would get guidance from the outset. Secondly, we anticipated that the Program would receive more proposals from a wider crosssection of schools and departments because of "viral" advertising among graduate students. Our experience was in line with our expectations.

At the same time, we saw challenges in addition to benefits from this approach. The first challenge was how to ensure fairness in the evaluation process. Most likely, a proposal written by seasoned faculty would be better quality and more compelling than one written by a student. To address this potential for disparity, we asked the proposals to identify clearly all the authors and their academic rank. We factored this information into the evaluation process, with student-written proposals getting more leeway.

Secondly, we were concerned that inexperienced students lacked the structure to put together a cohesive proposal that addressed all the necessary elements. To address this concern, we developed a numbered template for proposals and required that all proposals address the points in numbered sections of the proposal that related back to the solicitation. Not only did this ensure that all the necessary information was present in every proposal, it made evaluation a lot easier because of the inherent consistency of proposals.

\subsection{A lesson in following directions}

Since the solicitation specifically asked that proposals be three pages or less, and follow the given numbered format, we were rather surprised to see that a number of students (and faculty) chose not to follow directions. After tolerating this for the first few solicitations, we embarked on another teaching opportunity - emphasizing that when a solicitation specifies a format, requests specific information, or provides other directions, it is incumbent on the person preparing the proposal to follow those directions to the letter. The harsh reality of the real world is that reviewers do not have the time or motivation to go through the proposal and seek out the necessary information buried within. Rather, they are likely to mark that evaluation criterion with a low grade or as not responsive. This in turn, may significantly lower the proposal's probability of success. Given the low success percentage of many of today's solicitations, the reviewers' job often is to eliminate as many proposals as it is to pick successful ones.

We implemented this teaching opportunity in a manner that would not harm the authors. Rather than eliminating the proposal for failure to comply with the solicitation requirements of length and format, we acknowledged the receipt of the proposal and indicated that we had logged it in as received. We asked the author, however, to resubmit the proposal and this time to follow the directions. After we received the amended proposal, we sent another email explaining why we had subjected them to this extra effort, and what we hoped they had learned from the experience.

\subsection{Components for lab courses}

In the course of identifying interesting pre-commercial components, we came across one company that was discontinuing its line of tunable lasers. We were able to purchase a significant quantity of these lasers at deeply discounted prices. From previous solicitations, we were aware that researchers liked agile devices and that there would be high interest in these devices. The problem, however, was that these devices were truly pre-commercial, and as such, the company had not yet developed user manuals or data sheets and could provide only limited user information.

We turned this problem into a teaching opportunity. We provided Professor Ryszard Pryputniewicz at Worcester Polytechnic Institute about a dozen devices and all the information that we had - a couple of pages showing pin configuration and max/min parameters. Luckily, we also had a point of contact from the company who was willing to provide some additional information if so asked. Prof. Pryputniewicz took the tunable lasers and structured a design course around them. The students received a "black box" and their assignment was to characterize the device. After they had a thorough understanding of the device, the students designed an RS-232 interface/driver card for the laser. Lastly, they prepared a data package. They kindly made this package available to other groups who obtained the lasers through PTAP. 


\subsection{Devices for follow-on research}

Specialty fibers provided another interesting opportunity for using device availability to drive teaching opportunities. The cost of fabricating the glass preform and then drawing the fiber, set the fiber's cost. The cost is virtually the same whether a researcher needs 10 meters or 10,000 meters of fiber. Most researchers do not need the full output of a fiber draw, and therefore, the cost of a custom fiber draw is often prohibitive. PTAP has paid for specialty fiber draws and has delivered the requested length to researchers. PTAP then took the excess fiber, and after a negotiated lead-time, published the fiber specs on its web site and made it available to the research community at large. The benefits are two-fold: researchers get access to unusual fibers fabricated to their specifications, which are not available elsewhere. More importantly, once the original requestor publishes the experimental results, other researchers can obtain the same fiber to replicate the results and to perform follow-on work. In a way, PTAP acts as a consolidator without having to bring all the parties to the table at the outset.

\section{Conclusion}

The activities of PTAP demonstrate that program managers can create enhanced teaching opportunities through program is execution. Such activities enhance the value of the effort to the sponsors and participants. They also deliver content that ordinary classroom instruction cannot.

\section{Acknowledgements}

The National Science Foundation and the Defense Advanced Research Projects Agency fund PTAP and the author through NSF grant ECS-0630553. 